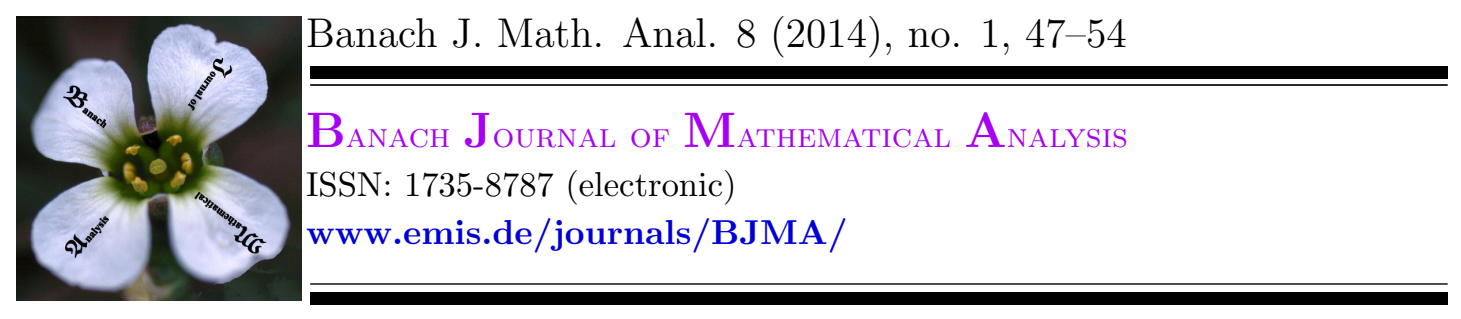

\title{
TENSOR PRODUCTS AND THE SPECTRAL CONTINUITY FOR $k$-QUASI- $*$-CLASS A OPERATORS
}

\author{
FUGEN GAO* AND XIAOCHUN LI \\ Communicated by M. Fujii
}

\begin{abstract}
An operator $T \in B(\mathcal{H})$ is called $k$-quasi-*-class A if $T^{* k}\left(\left|T^{2}\right|-\right.$ $\left.\left|T^{*}\right|^{2}\right) T^{k} \geq 0$ for a positive integer $k$, which is a common generalization of *-class A and quasi-*-class A. In this paper, firstly we prove some inequalities of this class of operators; secondly we consider the tensor products for $k$-quasi*-class A operators, giving a necessary and sufficient condition for $T \otimes S$ to be a $k$-quasi-*-class A operator when $T$ and $S$ are both non-zero operators; at last we prove that the spectrum is continuous on the class of all $k$-quasi- $*$-class A operators.
\end{abstract}

\section{INTRODUCTION AND PRELIMINARIES}

Let $\mathcal{H}$ be a separable complex Hilbert space and $\mathcal{C}$ be the set of complex numbers. Let $B(\mathcal{H})$ denote the $C^{*}$-algebra of all bounded linear operators acting on $\mathcal{H}$. If $T \in B(\mathcal{H})$, we shall write $\operatorname{ker} T$ and $\operatorname{ran} T$ for the null space and range of $T$ respectively. Also let $\alpha(T)=\operatorname{dim} \operatorname{ker} T, \beta(T)=\operatorname{dim} \operatorname{ker} T^{*}$ and let $\sigma(T), \sigma_{a}(T)$ denote the spectrum, approximate point spectrum of $T$. Let $p=p(T)$ be the ascent of $T$; i.e., the smallest nonnegative integer $p$ such that $\operatorname{ker} T^{p}=\operatorname{ker} T^{p+1}$. If such integer does not exist, we put $p(T)=\infty$. Analogously, let $q=q(T)$ be the descent of $T$; i.e., the smallest nonnegative integer $q$ such that $\operatorname{ran} T^{q}=\operatorname{ran} T^{q+1}$, and if such integer does not exist, we put $q(T)=\infty$. An operator $T \in B(\mathcal{H})$ is called upper (resp. lower) semi-Fredholm if $\operatorname{ran} T$ is closed and $\alpha(T)<\infty$ (resp. $\beta(T)<\infty)$. If $T \in B(\mathcal{H})$ is either an upper semi-Fredholm operator or a lower

Date: Received: 24 January 2013; Accepted: 16 March 2013.

* Corresponding author.

2010 Mathematics Subject Classification. Primary 47A63; Secondary 47B20.

Key words and phrases. $k$-quasi-*-class A, tensor product, spectral continuity. 
semi-Fredholm operator, then $T$ is called a semi-Fredholm operator, and the index of a semi-Fredholm operator $T \in B(\mathcal{H})$, denoted by $\operatorname{ind}(T)$, is given by the integer ind $(T)=\alpha(T)-\beta(T)$. If both $\alpha(T)$ and $\beta(T)$ are finite, then $T$ is called a Fredholm operator. An operator $T \in B(\mathcal{H})$ is called Weyl if it is Fredholm of index zero and Browder if it is Fredholm of finite ascent and descent. The essential spectrum $\sigma_{e}(T)$, the Weyl spectrum $\sigma_{w}(T)$ and the Browder spectrum $\sigma_{b}(T)$ of $T \in B(\mathcal{H})$ are defined by $\sigma_{e}(T)=\{\lambda \in \mathcal{C}: T-\lambda$ is not Fredholm $\}$, $\sigma_{w}(T)=\{\lambda \in \mathcal{C}: T-\lambda$ is not Weyl $\}$, and $\sigma_{b}(T)=\{\lambda \in \mathcal{C}: T-\lambda$ is not Browder $\}$.

Let $\mathcal{H}, \mathcal{K}$ be complex Hilbert spaces and $\mathcal{H} \otimes \mathcal{K}$ the tensor product of $\mathcal{H}, \mathcal{K}$; i.e., the completion of the algebraic tensor product of $\mathcal{H}, \mathcal{K}$ with the inner product $\left\langle x_{1} \otimes y_{1}, x_{2} \otimes y_{2}\right\rangle=\left\langle x_{1}, x_{2}\right\rangle\left\langle y_{1}, y_{2}\right\rangle$ for $x_{1}, x_{2} \in \mathcal{H}, y_{1}, y_{2} \in \mathcal{K}$. Let $T \in B(\mathcal{H})$ and $S \in B(\mathcal{K}) . T \otimes S \in B(\mathcal{H} \otimes \mathcal{K})$ denotes the tensor product of $T$ and $S$; i.e., $(T \otimes S)(x \otimes y)=T x \otimes S y$ for $x \in \mathcal{H}, y \in \mathcal{K}$.

Recall that $T \in B(\mathcal{H})$ is called $p$-hyponormal for $p>0$ if $\left(T^{*} T\right)^{p}-\left(T T^{*}\right)^{p} \geq$ 0 [10]; when $p=1, T$ is called hyponormal. And $T$ is called paranormal if $\|T x\|^{2} \leq\left\|T^{2} x\right\|\|x\|$ for all $x \in \mathcal{H}[10,11]$. And $T$ is called normaloid if $\left\|T^{n}\right\|=\|T\|^{n}$ for all $n \in \mathbb{N}$ (equivalently, $\|T\|=r(T)$, the spectral radius of $T$ ). In order to discuss the relations between paranormal and $p$-hyponormal and loghyponormal operators ( $T$ is invertible and $\log T^{*} T \geq \log T T^{*}$ ), Furuta, Ito and Yamazaki [12] introduced a very interesting class of operators: class A defined by $\left|T^{2}\right|-|T|^{2} \geq 0$, where $|T|=\left(T^{*} T\right)^{\frac{1}{2}}$ which is called the absolute value of $T$ and they showed that class A is a subclass of paranormal and contains $p$-hyponormal and log-hyponormal operators. Recently Duggal, Jeon and Kim [7] introduced *-class A(i.e., $\left.\left|T^{2}\right|-\left|T^{*}\right|^{2}\right) \geq 0$ ) operators.

Definition 1.1. $T \in B(\mathcal{H})$ is called a $k$-quasi-*-class A operator for a positive integer $k$ if

$$
T^{* k}\left(\left|T^{2}\right|-\left|T^{*}\right|^{2}\right) T^{k} \geq 0
$$

when $k=1$, called quasi-*-class A operator, see [25].

For more interesting properties on $k$-quasi-*-class A operators, see [7, 21, 22]. It is clear that

the class of $*$-class A operators $\subseteq$ the class of quasi-*-class A operators $\subseteq$ the class of $k$-quasi- $*$-class A operators

and

the class of $k$-quasi- $*$-class $\mathrm{A}$ operators $\subseteq$ the class of $(k+1)$-quasi- $*$-class $\mathrm{A}$ operators.

We show that the inclusion relations above are strict, by an example which appeared in [13].

Example 1.2. Given a bounded sequence of positive numbers $\left\{\alpha_{i}\right\}_{i=0}^{\infty}$. Let $T$ be the unilateral weighted shift operator on $l^{2}$ with the canonical orthonormal basis $\left\{e_{n}\right\}_{n=0}^{\infty}$ by $T e_{n}=\alpha_{n} e_{n+1}$ for all $n \geq 0$, that is, 


$$
T=\left(\begin{array}{ccccc}
0 & & & & \\
\alpha_{0} & 0 & & & \\
& \alpha_{1} & 0 & & \\
& & \alpha_{2} & 0 & \\
& & & \ddots & \ddots
\end{array}\right)
$$

Straightforward calculations show that $T$ is a $k$-quasi-*-class A operator if and only if $\alpha_{n} \alpha_{n+1} \geq \alpha_{n-1}^{2}$ for all $n \geq k$. So if $\alpha_{k} \alpha_{k+1}<\alpha_{k-1}^{2}$ and $\alpha_{n} \alpha_{n+1} \geq \alpha_{n-1}^{2}$ for all $n \geq k+1$, then $T$ is a $(k+1)$-quasi-*-class A operator, but not a $k$-quasi- $*$-class A operator.

In this paper, firstly we prove some inequalities of this class of operators; secondly we consider the tensor products for $k$-quasi-*-class A operators, giving a necessary and sufficient condition for $T \otimes S$ to be a $k$-quasi-*-class A operator when $T$ and $S$ are both non-zero operators; at last we prove that the spectrum is continuous on the class of all $k$-quasi-*-class A operators.

\section{TEnSOR PRODUCTS FOR $k$-QUASI- $*$-CLASS A OPERATORS}

At first, we shall prove some inequalities of $k$-quasi-*-class A operators.

Theorem 2.1. Let $T \in B(\mathcal{H})$ be a $k$-quasi-*-class $A$ operator for a positive integer $k$. Then the following assertions hold.

(1) $\left\|T^{n+2} x\right\|\left\|T^{n} x\right\| \geq\left\|T^{*} T^{n} x\right\|^{2}$ for all $x \in \mathcal{H}$ and all positive integers $n \geq k$.

(2) If $\bar{T}^{n}=0$ for some positive integer $n \geq k$, then $T^{k}=0$.

Proof. (1) Since it is clear that $k$-quasi-*-class A operators are $(k+1)$-quasi-*-class A operators, we only need to prove the case $n=k$. Since

$$
\left\langle T^{* k}\left|T^{*}\right|^{2} T^{k} x, x\right\rangle=\left\langle T^{* k} T T^{*} T^{k} x, x\right\rangle=\left\|T^{*} T^{k} x\right\|^{2},
$$

and

$$
\left\langle T^{* k}\left|T^{2}\right| T^{k} x, x\right\rangle=\left\langle\left|T^{2}\right| T^{k} x, T^{k} x\right\rangle \leq\left\|\left|T^{2}\right| T^{k} x\right\|\left\|T^{k} x\right\|=\left\|T^{k+2} x\right\|\left\|T^{k} x\right\| .
$$

We have that

$$
\left\|T^{k+2} x\right\|\left\|T^{k} x\right\| \geq\left\|T^{*} T^{k} x\right\|^{2}
$$

for all $x \in \mathcal{H}$ for $T$ is a $k$-quasi- $*$-class A operator.

(2) If $n=k$, it is obvious that $T^{k}=0$. If $T^{k+1}=0$, then $T^{k+2}=0$. So we have that $T^{*} T^{k}=0$ by (1). Hence we have that $\left\|T^{k} x\right\|^{2}=\left\langle T^{*} T^{k} x, T^{k-1} x\right\rangle=0$ for all $x \in \mathcal{H}$. So $T^{k}=0$. The rest of the proof is similar.

Let $T \otimes S$ denote the tensor product on the product space $\mathcal{H} \otimes \mathcal{K}$ for nonzero $T \in B(\mathcal{H})$ and $S \in B(\mathcal{K})$. The operation of taking tensor products $T \otimes S$ preserves many properties of $T \in B(\mathcal{H})$ and $S \in B(\mathcal{K})$, but by no means all 
of them. For example the normaloid property is invariant under tensor products, the spectraloid property is not (see [23] pp. 623 and 631); and $T \otimes S$ is normal if and only if $T$ and $S$ are normal [16, 26]; however there exist paranormal operators $T \in B(\mathcal{H})$ and $S \in B(\mathcal{K})$ such that $T \otimes S$ is not paranormal [1]. Duggal [6] showed that for non-zero $T \in B(\mathcal{H})$ and $S \in B(\mathcal{K}), T \otimes S$ is $p$-hyponornal if and only if $T, S$ are $p$-hyponornal. This result was extended to p-quasihyponormal operators, class A operators, log-hyponormal operators and class $\mathrm{A}(s, t)$ operators $\left(\left(\left|T^{*}\right|^{t}|T|^{2 s}\left|T^{*}\right|^{t}\right)^{\frac{t}{s+t}} \geq\left|T^{*}\right|^{2 t}, s, t>0\right)$ in [19, 20, 27], respectively. The following theorem gives a necessary and sufficient condition for $T \otimes S$ to be a $k$-quasi- $*$-class A operator when $T$ and $S$ are both non-zero operators, which is an extension of [7] Theorem 3.2.

Theorem 2.2. Let $T \in B(\mathcal{H})$ and $S \in B(\mathcal{K})$ be non-zero operators. Then $T \otimes S \in B(\mathcal{H} \otimes \mathcal{K})$ is a $k$-quasi-*-class $A$ operator if and only if one of the following assertions holds:

(1) $T^{k}=0$ or $S^{k}=0$.

(2) $T$ and $S$ are $k$-quasi-*-class $A$ operators.

Proof. It is clear that $T \otimes S$ is a $k$-quasi-*-class A operator if and only if

$$
\begin{aligned}
& (T \otimes S)^{* k}\left(\left|(T \otimes S)^{2}\right|-\left|(T \otimes S)^{*}\right|^{2}\right)(T \otimes S)^{k} \geq 0 \\
& \Longleftrightarrow T^{* k}\left(\left|T^{2}\right|-\left|T^{*}\right|^{2}\right) T^{k} \otimes S^{* k}\left|S^{2}\right| S^{k}+T^{* k}\left|T^{*}\right|^{2} T^{k} \otimes S^{* k}\left(\left|S^{2}\right|-\left|S^{*}\right|^{2}\right) S^{k} \geq 0 \\
& \Longleftrightarrow T^{* k}\left|T^{2}\right| T^{k} \otimes S^{* k}\left(\left|S^{2}\right|-\left|S^{*}\right|^{2}\right) S^{k}+T^{* k}\left(\left|T^{2}\right|-\left|T^{*}\right|^{2}\right) T^{k} \otimes S^{* k}\left|S^{*}\right|^{2} S^{k} \geq 0 .
\end{aligned}
$$

Therefore the sufficiency is clear.

To prove the necessary. Suppose that $T \otimes S$ is a $k$-quasi-*-class A operator. Let $x \in \mathcal{H}$ and $y \in \mathcal{K}$ be arbitrary. Then we have

$$
\left\langle T^{* k}\left(\left|T^{2}\right|-\left|T^{*}\right|^{2}\right) T^{k} x, x\right\rangle\left\langle S^{* k}\left|S^{2}\right| S^{k} y, y\right\rangle+\left\langle T^{* k}\left|T^{*}\right|^{2} T^{k} x, x\right\rangle\left\langle S^{* k}\left(\left|S^{2}\right|-\left|S^{*}\right|^{2}\right) S^{k} y, y\right\rangle \geq 0 .
$$

It suffices to prove that if (1) does not hold, then (2) holds. Suppose that $T^{k} \neq 0$ and $S^{k} \neq 0$. To the contrary, assume that $T$ is not a $k$-quasi-*-class A operator, then there exists $x_{0} \in \mathcal{H}$ such that

$$
\left\langle T^{* k}\left(\left|T^{2}\right|-\left|T^{*}\right|^{2}\right) T^{k} x_{0}, x_{0}\right\rangle=\alpha<0 \text { and }\left\langle T^{* k}\left|T^{*}\right|^{2} T^{k} x_{0}, x_{0}\right\rangle=\beta>0 .
$$

From (2.1) we have

$$
\alpha\left\langle S^{* k}\left|S^{2}\right| S^{k} y, y\right\rangle+\beta\left\langle S^{* k}\left(\left|S^{2}\right|-\left|S^{*}\right|^{2}\right) S^{k} y, y\right\rangle \geq 0
$$

for all $y \in \mathcal{K}$, that is,

$$
(\alpha+\beta)\left\langle S^{* k}\left|S^{2}\right| S^{k} y, y\right\rangle \geq \beta\left\langle S^{* k}\left|S^{*}\right|^{2} S^{k} y, y\right\rangle
$$

for all $y \in \mathcal{K}$. Therefore $S$ is a $k$-quasi-*-class A operator. We have

$$
\left\langle S^{* k}\left|S^{*}\right|^{2} S^{k} y, y\right\rangle=\left\|S^{*} S^{k} y\right\|^{2} \text { and }\left\langle S^{* k}\left|S^{2}\right| S^{k} y, y\right\rangle \leq\left\|S^{k+2} y\right\|\left\|S^{k} y\right\| \text {. }
$$

So we have

$$
(\alpha+\beta)\left\|S^{k+2} y\right\|\left\|S^{k} y\right\| \geq \beta\left\|S^{*} S^{k} y\right\|^{2}
$$


for all $y \in \mathcal{K}$ by (2.2). Because $S$ is a $k$-quasi-*-class A operator, from [21] Lemma 2.1 we can write $S=\left(\begin{array}{ll}S_{1} & S_{2} \\ 0 & S_{3}\end{array}\right) \quad$ on $\mathcal{K}=\overline{\operatorname{ran}\left(S^{k}\right)} \bigoplus \operatorname{ker} S^{* k}$, where $S_{1}$ is a *-class A operator (hence it is normaloid by [21] Theorem 2.6). By (2.3) we have

$$
(\alpha+\beta)\left\|S_{1}^{2} \eta\right\|\|\eta\| \geq \beta\left\|S^{*} \eta\right\|^{2} \geq \beta\left\|S_{1}^{*} \eta\right\|^{2} \text { for all } \eta \in \overline{\operatorname{ran}\left(S^{k}\right)} .
$$

So we have

$$
(\alpha+\beta)\left\|S_{1}\right\|^{2}=(\alpha+\beta)\left\|S_{1}^{2}\right\| \geq \beta\left\|S_{1}^{*}\right\|^{2}=\beta\left\|S_{1}\right\|^{2},
$$

where equality holds since $S_{1}$ is normaloid.

This implies that $S_{1}=0$. Since $S^{k+1} y=S_{1} S^{k} y=0$ for all $y \in \mathcal{H}$, we have $S^{k+1}=0$. Since $S$ is a $k$-quasi-*-class A operator, by Theorem 2.1 (ii) we have that $S^{k}=0$. This contradicts the assumption $S^{k} \neq 0$. Hence $T$ must be a $k$-quasi*-class A operator. A similar argument shows that $S$ is also a $k$-quasi-*-class A operator. The proof is complete.

\section{Spectral CONTINUITy FOR $k$-QUASI-*-Class A Operators}

Let $\left\{\tau_{n}\right\}$ be a sequence of compact subsets of $\mathcal{C}$. Then its limit inferior is defined by

$$
\lim \inf \left\{\tau_{n}\right\}=\left\{\lambda \in \mathcal{C}: \text { there exists } \lambda_{n} \in \tau_{n} \text { such that } \lambda_{n} \longrightarrow \lambda\right\}
$$

and its limit superior is defined by

$$
\lim \sup \left\{\tau_{n}\right\}=\left\{\lambda \in \mathcal{C}: \text { there exists } \lambda_{n_{k}} \in \tau_{n_{k}} \text { such that } \lambda_{n_{k}} \longrightarrow \lambda\right\} .
$$

If $\lim \inf \left\{\tau_{n}\right\}=\lim \sup \left\{\tau_{n}\right\}$, then $\lim \left\{\tau_{n}\right\}$ is defined by this common limit. A map $p$, defined on $B(\mathcal{H})$, whose values are compact subsets of $\mathcal{C}$, is said to be upper (resp. lower) semi-continuous at $T$, if $T_{n} \longrightarrow T$ then $\lim \sup p\left(T_{n}\right) \subset p(T)$ (resp. $p(T) \subset \lim \inf p\left(T_{n}\right)$. If $p$ is both upper and lower semi-continuous at $T$, then it is said to be continuous at $T$ and in this case $\lim p\left(T_{n}\right)=p(T)$.

For every $T \in B(\mathcal{H}), \sigma(T)$ is a compact subset of $\mathcal{C}$. The function $\sigma$ viewed as a function from $B(\mathcal{H})$ into the set of all compact subsets of $\mathcal{C}$, equipped with the Hausdorff metric, is well known to be upper semi-continuous, but fails to be continuous in general. Conway and Morrel [3] have carried out a detailed study of spectral continuity in $B(\mathcal{H})$. Recently, the continuity of spectrum was considered when restricted to certain subsets of the entire manifold of Toeplitz operators in $[9,17]$. It has been proved that $\sigma$ is continuous in the set of normal operators and hyponormal operators in [15]. And this result has been extended to quasihyponormal operators by S. V. Djordjević in [4], to $p$-hyponormal operators by Hwang and Lee in [18], to $(p, k)$-quasihyponormal, M-hyponormal, *-paranormal and paranormal operators by Duggal, Jeon and Kim in [8], and to quasi-class $(A, k)$ operators by Gao and Fang in [14]. In the following, we extend this result to $k$-quasi-*-class A operators. 
In the following, we prove that spectrum $\sigma$ is continuous on the set of all $k$-quasi-*-class A operators.

Lemma 3.1. Let $T$ be a $k$-quasi-*-class $A$ operator for a positive integer $k$. Then the following assertions hold:

(1) If $T$ is quasinilpotent (i.e., $\sigma(T)=\{0\}$ ), then $T$ is nilpotent.

(2) For every non-zero $\lambda \in \sigma_{p}(T)$, the matrix representation of $T$ with respect to the decomposition $\mathcal{H}=\operatorname{ker}(T-\lambda) \bigoplus(\operatorname{ker}(T-\lambda))^{\perp}$ is: $T=\left(\begin{array}{ll}\lambda & 0 \\ 0 & B\end{array}\right)$ for some operator $B$ satisfying $\lambda \notin \sigma_{p}(B)$ and $\sigma(T)=\{\lambda\} \cup \sigma(B)$.

Proof. Suppose $T$ is a $k$-quasi-*-class A operator for a positive integer $k$. (1) holds by [22] Corollary 2.2. If $\lambda \neq 0$ and $\lambda \in \sigma_{p}(T)$, we have that $\operatorname{ker}(T-\lambda)$ reduces $T$ by [21] Lemma 2.5. So we have that $T=\left(\begin{array}{cc}\lambda & 0 \\ 0 & B\end{array}\right)$ on $\mathcal{H}=\operatorname{ker}(T-\lambda) \bigoplus(\operatorname{ker}(T-$ $\lambda))^{\perp}$ for some operator $B$ satisfying $\lambda \notin \sigma_{p}(B)$ and $\sigma(T)=\{\lambda\} \cup \sigma(B)$.

The Berberian extension theorem shows that given an operator $T \in B(\mathcal{H})$, there exists a Hilbert space $\mathcal{K} \supseteq \mathcal{H}$ and an isometric $*$-isomorphism $T \rightarrow T^{\circ} \in$ $B(\mathcal{K})$ preserving order such that $\sigma(T)=\sigma\left(T^{\circ}\right)$ and $\sigma_{p}\left(T^{\circ}\right)=\sigma_{a}\left(T^{\circ}\right)=\sigma_{a}(T)$. See details in the following lemma:

Lemma 3.2. [2]. Let $\mathcal{H}$ be a complex Hilbert space. Then there exists a Hilbert space $\mathcal{K}$ such that $\mathcal{H} \subset \mathcal{K}$ and a map $\varphi: B(\mathcal{H}) \longrightarrow B(\mathcal{K})$ such that

(1) $\varphi$ is a faithful $*$-representation of the algebra $B(\mathcal{H})$ on $\mathcal{K}$, i.e., $\varphi(S+T)=$ $\varphi(S)+\varphi(T), \varphi(\lambda T)=\lambda \varphi(T), \varphi(S T)=\varphi(S) \varphi(T), \varphi\left(T^{*}\right)=(\varphi(T))^{*}$, $\varphi(I)=I$ and $\|\varphi(T)\|=\|T\|$ for any $S, T \in B(\mathcal{H})$ and $\lambda \in \mathcal{C}$.

(2) $\varphi(A) \geq 0$ for any $A \geq 0$ in $B(\mathcal{H})$.

(3) $\sigma_{a}(T)=\sigma_{a}(\varphi(T))=\sigma_{p}(\varphi(T))$ for any $T \in B(\mathcal{H})$.

Theorem 3.3. The spectrum $\sigma$ is continuous on the set of $k$-quasi-*-class $A$ operators for a positive integer $k$.

Proof. Suppose $T$ is a $k$-quasi-*-class A operator for a positive integer $k$. Let $\varphi: B(\mathcal{H}) \longrightarrow B(\mathcal{K})$ be Berberian's faithful $*$-representation of Lemma 3.2. In the following, we shall show that $\varphi(T)$ is also a $k$-quasi-*-class A operator for a positive integer $k$. In fact, since $T$ is a $k$-quasi-*-class $\mathrm{A}$ operator, we have $T^{* k}\left(\left|T^{2}\right|-\left|T^{*}\right|^{2}\right) T^{k} \geq 0$. Hence we have

$$
\begin{aligned}
& (\varphi(T))^{* k}\left(\left|(\varphi(T))^{2}\right|-\left|\varphi(T)^{*}\right|^{2}\right)(\varphi(T))^{k} \\
& =\varphi\left(T^{* k}\left(\left|T^{2}\right|-\left|T^{*}\right|^{2}\right) T^{k}\right) \text { by Lemma } 3.2(1) \\
& \geq 0 \text { by Lemma } 3.2(2) .
\end{aligned}
$$

So we have that its Berberian extension $T^{\circ}=\varphi(T)$ is also a $k$-quasi- $*$-class $\mathrm{A}$ operator for a positive integer $k$. By Lemma 3.1 we have that $T$ belongs to the set $\mathcal{C}(i)$ (see definition in [8]). So we have that the spectrum $\sigma$ is continuous on the set of $k$-quasi-*-class A operators for a positive integer $k$ by [8] Theorem 1.1. This completes the proof. 
Corollary 3.4. The Weyl spectrum $\sigma_{w}$ is continuous if and only if the Browder spectrum $\sigma_{b}$ is continuous on the set of $k$-quasi-*-class $A$ operators for a positive integer $k$.

Proof. Suppose $T$ is a $k$-quasi-*-class A operator for a positive integer $k$. By Theorem 3.3 and [24] Theorem 2.2, we have that Browder's theorem holds for $T$. Hence Corollary 3.4 holds by the remark of [24] or the equivalence between (ii) and (iii) of [5] Theorem 2.2.

Acknowledgement. We would like to express our cordial gratitude to the referee for his valuable advice and suggestion. This work was supported by the National Natural Science Foundation of China (11071188), (11271112); the Natural Science Foundation of the Department of Education, Henan Province (2011A110009), (13B110077); the Youth Science Foundation of Henan Normal University and the New Teachers Science Foundation of Henan Normal University (No. qd12102) and Key Scientific and Technological Project of Henan Province (122102210132).

\section{REFERENCES}

1. T. Ando, Operators with a norm condition, Acta Sci. Math. (Szeged) 33 (1972), 169-178.

2. S.K. Berberian, Approximate proper vectors, Proc. Amer. Math. Soc. 13 (1962), 111-114.

3. J.B. Conway and B.B. Morrel, Operators that are points of spectral continuity, Integral Equation Operator Theory 2 (1979), 174-198.

4. S. V. Djordjević, Continuity of the essential spectrum in the class of quasihyponormal operators, Vesnik Math. 50 (1998), 71-74.

5. S.V. Djordjević and Y.M. Han, Browder's theorem and spectral continuity, Glasgow Math. J. 42 (2000), 479-486.

6. B.P. Duggal, Tensor products of operators-strong stability and p-hyponormality, Glasg. Math. J. 42 (2000), 371-381.

7. B.P. Duggal, I.H. Jeon and I.H. Kim, On *-paranormal contractions and properties for *-class A operators, Linear Algebra Appl. 436 (2012), 954-962.

8. B.P. Duggal, I.H. Jeon and I.H. Kim, Continuity of the spectrum on a class of upper triangular operator matrices, J. Math. Anal. Appl. 370 (2010), 584-587.

9. D.R. Farenick and W.Y. Lee, Hyponormality and spectra of Toeplitz operators, Trans. Amer. Math. Soc. 348 (1996), 4153-4174.

10. T. Furuta, On the class of paranormal operators, Proc. Japan Acad. 43 (1967), 594-598.

11. T. Furuta, Invitation to Linear Operators, Taylor and Francis, London, 2001.

12. T. Furuta, M. Ito and T. Yamazaki, A subclass of paranormal operators including class of log-hyponormal and several classes, Sci. Math. 1 (1998), no. 3, 389-403.

13. F.G. Gao, and X.C. Fang, On k-quasi-class A operators, J. Inequal. Appl. 2009 (2009), Article ID 921634, 1-10.

14. F.G. Gao, and X.C. Fang, Generalized Weyl's theorem and spectral continuity for quasiclass $(A, k)$ operators, Acta Sci. Math. (Szeged) $\mathbf{7 8}$ (2012), 241-250.

15. P.R. Halmos, A Hilbert Space Problem Book, Springer-Verlag, New York, 1982.

16. J.C. Hou, On tensor products of operators, Acta Math. Sin. (N. S.) 9 (1993), 195-202.

17. I.S. Hwang and W.Y. Lee, On the continuity of spectra of Toeplitz operators, Arch. Math. 70 (1998), 66-73.

18. I.S. Hwang and W.Y. Lee, The spectrum is continuous on the set of p-hyponormal operators, Math. Z. 235 (2000), 151-157.

19. I.H. Jeon and B.P. Duggal, On operators with an absolute value condition, J. Korean. Math. Soc. 41 (2004), 617-627. 
20. I.H. Kim, Tensor products of log-hyponormal operators, Bull. korean math. Soc. 42 (2005), 269-277.

21. S. Mecheri, Isolated points of spectrum of k-quasi-*-class A operators, Studia Math. 208 (2012), 87-96.

22. S. Mecheri, On operators satisfying an inequality, J. Inequal. Appl. 2012 2012:244 doi:10.1186/1029-242X-2012-244.

23. T. Saitô, Hyponormal Operators and Related Topics, Lecture Notes in Mathematics, 247, Springer-Verlag, 1971.

24. S. Sánchez-Perales and S. V. Djordjević, Continuity of spectra and compact perturbations, Bull. Korean Math. Soc. 48 (2011), 1261-1270.

25. J.L. Shen, F. Zuo and C.S. Yang, On operators satisfying $T^{*}\left|T^{2}\right| T \geq T^{*}\left|T^{*}\right|^{2} T$, Acta Math. Sinica (English Ser.) 26 (2010), 2109-2116.

26. J. Stochel, Seminormality of operators from their tensor products, Proc. Amer. Math. Soc. 124 (1996), 435-440.

27. K. Tanahashi, Tensor products of log-hyponormal operators and of class A(s,t) operators, Glasgow Math. J. 46 (2004), 91-95.

College of Mathematics and Information Science, Henan Normal University, Xinxiang, Henan, 453007, P.R. China.

E-mail address: gaofugen08@126.com

E-mail address: 1.xiaochun@tom.com 